\title{
EDITORIAL
}

\section{Low cholesterol and violent death}

\section{The evidence, the gaps, the theory and the practical implications}

Ronán M Conroy, Department of Epidemiology and Preventive Medicine, Royal College of Surgeons in Ireland, Mercer Building, Dublin 2, Ireland.

Key Words: Accidents; Cholesterol; Depression; Hostility; Serotonin; Suicide.

\section{The Problem}

The early trials of cholesterol-lowering drugs showed a promising reduction of cardiovascular mortality associated with treatment. Disappointingly, though, overall mortality was frequently not changed, due to an increase in non-cardiovascular deaths. In particular, violent death (suicide, murder and accidents) was increased in those who had received intervention. Some investigators expressed the hope that this was simply an unfortunate coincidence, but by the time Matthew Muldoon (I) reviewed the literature in 1990, the association had occurred in too many trials to be just chance. Furthermore, the effect didn't seem to depend on what drug was used to lower cholesterol; drugs with widely-differing modes of action all seemed to share a common effect. At the time of the publication of the Lipid Research Clinics Trial (2), the Finnish researcher, Matti Virkkunen (3) proposed that the excess of violent deaths in those treated with cholestyramine might be connected to findings he had made linking low cholesterol and low impulse control, though a mechanism by which this happened was far from obvious.

\section{The Evidence}

Concern about a link between low cholesterol and violent death lead investigators to examine data from prospective studies. Juha Pekkanen and his colleagues analysed 25-year follow-up data on the Finnish cohort of the Seven Countries Study, and found no relationship between low serum cholesterol and violent death (4). They suggested, amongst other possible explanations, that the associations found by others between low serum cholesterol and poor impulse control could be due to low serotonin turnover. Low serotonin activity (usually measured as cerebrospinal 5-HIAA) had been linked to suicidal behaviour since 1976, when Asberg and colleagues reported on suicidal behaviour in a group of depressed patients (5). By the time an anonymous editorial in the Lancet reviewed the topic in 1987, low serotonin turnover had been obversed in a wide variety of disorders characterised by low impulse control(6). Pekkanen and his colleagues suggested that low serotonin turnover in the brain could result in enhanced insulin secretion which, in turn, could lower cholesterol levels, though this has yet to be substantiated by research.

One study which found a link between low serum cholesterol on a single measurement and subsequent risk of violent death was a report in Lindberg et al (7) who analysed data from over 50,000 men and women who were being followed up by the Värmland study in Sweden. They found that men with a low serum cholesterol $<5.3 \mathrm{mmol} / \mathrm{L}(205 \mathrm{mg} \%$ ) had an increased risk of violent death in the first six years of the 20-year follow-up period. There was no relationship between low cholesterol and risk subsequently, and the relationship did not emerge in women. Other attempts to link low cholesterol to subsequent violent death in followup studies came to negative conclusions, including an analysis of all sixteen cohorts of the Seven Countries Study (8). However, a major review, co-ordinated by the National Heart, Lung and Blood Institute, which pooled data from almost 125,000 people in 19 cohort studies from the United States, Europe, Israel and Japan found that the risk ratio for violent death occurring more than 5 years from baseline cholesterol measurement was 3.0 in men and 1.26 in women whose cholesterol was $<4.2 \mathrm{mmol} / \mathrm{L}(<160 \mathrm{mg} \%$ ) (9). The reason for their exclusion of deaths occurring in the first five years of follow-up was to exclude those whose cholesterol had been lowered by disease. In a recently-published report on 28-year follow-up of almost 3,000 Dutch civil servants who had their cholesterol measured in 1953 and 1954, Albertine Schuit and her co-authors reported that, adjusted for age and sex, those in the lowest tertile of cholesterol had a 2.4-fold increased risk of violent death over the 28-year period (10). The effects of adjusting for smoking and alcohol consumption were negligible.

Some surveys of coronary risk factors also measured personality characteristics, and researchers examined these to see if hostility, aggressiveness or other relevant traits were associated with cholesterol. The Whitehall study found no relationship between cholesterol and hostility (11), and an analysis of those with low

Editor in Chief: Mark Hartman (Dublin). Editors: Timothy Dinan (London), Roy McClelland (Belfast). Deputy Editor: Brian O'Shea (Dublin). Associate Editors: Ken Brown (Belfast), Patricia Casey (Dublin), Anthony Clare (Dublin), Stephen Cooper (Belfast), Thomas Fahy (Galway), Michael Fitzgerald (Dublin), Michael Kelleher (Cork), David King (Belfast), Brian Leonard (Galway), Aidan McGennis (Dublin), Ciaran O'Boyle (Dublin), Eadbhard O'Callaghan (Dublin), Art O'Connor (Dublin), Ethna O'Gorman (Belfast), lan Pullen (Edinburgh), David Sheehan (Tampa), Philip Snaith (Leeds), Hugh Staunton (Dublin), John Waddington (Dublin), Richard Williams (Calgary). Statistical Editor: Leslie Daly (Dublin). Deputy Statistical Editor: Ronan Conroy (Dublin). 
cholesterol suggested that other factors associated with low cholesterol were in fact responsible for the association with violent death. In a surprising finding, however, the authors of the Edinburgh Artery Study reported that, corrected for other lipids and risk factors, serum triglycerides were associated with hostile thoughts and acts and with denigratory attitudes towards others in men. In women, only the latter variable had an independent association with triglycerides. This finding remains unexplained, though I have suggested that, since low-fat cholesterol-lowering diets are associated with a reduction in cholesterol but not in triglycerides (12), the association between triglycerides and hostility which emerged when the analysis was corrected for absolute cholesterol level might have been the result of hostility being related not to low cholesterol but to lowered cholesterol (13). In another study which related lipids to depression, Timo Strandberg reported that in the Helsinki Ageing Study both low HDL cholesterol and high triglycerides were linked to depression, assessed using the Zung SDS scale (14). There was no relationship between low cholesterol and depression in either sex, but their results, added to those of Fowkes, suggest that the relationship of mood to triglycerides needs further investigation.

\section{The Theory}

How could lowering cholesterol produce changes at the neurological level which increased risk violent death? Preston Mason, reviewing the data on serum cholesterol and neurological function, pointed out that there were two important questions to be answered: does altering blood cholesterol result in alterations in brain cholesterol, and does altering brain cholesterol alter neurological function (15). While neither question has been fully explored, he concluded that the current balance of evidence was that the answer to both questions was yes. Concentrations of cholesterol in nerve cell membranes appear highly influenced by the cholesterol content of the surrounding medium. Increases or decreases in nerve-cell membrane cholesterol, in turn, have been shown to disturb the function of cell membrane proteins, including ion channels and neurotransmitter receptor binding. Hyman Engelberg (16) posited a specific mechanism linking cholesterol to violent death: he pointed out that in animal studies, decreased brain synaptosomal membrane cholesterol is associated with decreased numbers of serotonin receptors. Membrane cholesterol exchanges freely with cholesterol in the surrounding medium, so lowering serum cholesterol concentration could lower brain serotonin turnover. Low cholesterol is also associated with higher serotonin reuptake velocity at platelet level (17), and either or both of these two factors could provide a mechanism by which lowered cholesterol could decrease the activity of brain serotonergic systems. Though serotonergic activity is only one of a number of effects that may be produced by lowered cholesterol, the current elucidation of the role which serotonin plays in the nervous system makes it a likely candidate for the pathway between cholesterol and violent death. In an extensive review of the modulatory role of serotonin in neural information processing, Michele Spoont proposed that serotonergic circuits operate to inhibit information flow (18). Alterations in serotonin activity influence behavioural responses to both internal and external stimuli; in particular, the exaggeration of signal saliency that would result from serotonergic dysfunction would have the effect of reducing the importance of context cues such as social appropriateness, producing a behavioural picture characterised by impulsiveness in which actions were determined by the most obvious aspects of the situation without giving due weight to more subtle factors such as long-term consequences. This, taken with the evidence linking reduced serotonergic function to aggression and low impulse control, found in a wide variety of abnormal psychiatric conditions, suggests that the link between low cholesterol and violent death was a causal one.

Apart from suicide, poor impulse control could contribute to fatal accidents by lowering the person's ability to choose the appropriate response from a set of competing responses or by increasing the amount of time needed to respond appropriately - as, for example, when a motorist is faced with the choice of swerving or braking. Individuals with low impulse control may also provoke aggression more easily, resulting in an increase of deaths by murder. Although lower serotonin metabolite levels have been demonstrated in individuals with disorders characterised by impulsivity, the available human evidence still rests on the study of such cases, rather than on studies of the general population.

Aside from it's rôle in impulse control, serotonin is also implicated in major depressive disorder, and this could provide a second pathway between cholesterol and suicide, since major depressive disorder frequently involved ideas of guilt or worthlessness, combined with suicidal ideation. Recent corroborative evidence has emerged from the report of Morgan (19) and his colleagues that in older men low cholesterol is associated with depression, as measured by the Beck Depression Inventory. Unfortunately, they had no data on women. Although their sample was criticized for being unrepresentative, the authors subsequently argued (20) that there is no reason to assume that the associations they found are confined to the group they studied. They reported that the odds ratio for depression (defined as a score of 13 or more on the Beck Depression Inventory) was $2.5(95 \%$ CI 1.1 to 5.8$)$ in men aged over 60 when logistic regression was used to adjust for the confounding effects of age, number of chronic diseases, number of medications, perceived health status, perceived physical functioning and weight (weight loss was not associated with depression). This provides further evidence that the association is not spurious.

A subsequent report from the Helsinki Ageing Study found no association between depression, assessed using the Zung scale, and cholesterol (2I), while a French research group from the EVA longitudinal study on cardiovascular and cognitive ageing found that those in the lowest cholesterol quintile had a more-than-twofold increase in prevalence of depression, which they assessed using the CES-D scale (22). This increased prevalence was independent of both the use of cholesterol-lowering and lipid-lowering drugs. There have also been recent case reports of depressive symptoms following the use of cholesterol-lowering drugs (23), though as yet no formal prospective study has been carried out. 
A new kind of anxiety therapy has arrived

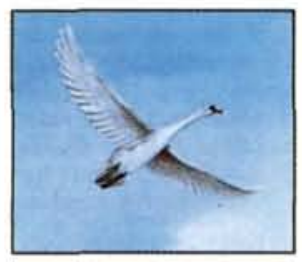

$\square$ Unrelated to the benzodiazepines or other anxiety therapies ${ }^{1}$

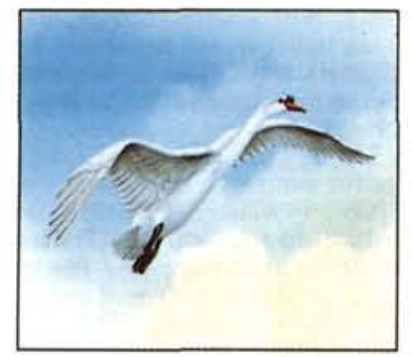

As effective as the benzodiazepines in relieving anxiety ${ }^{2}$

No evidence of dependence or abuse potential $^{3}$

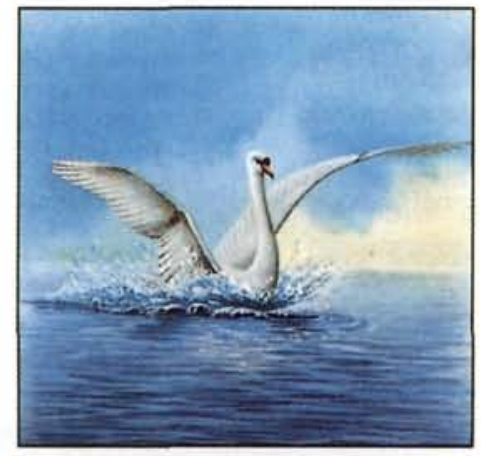

$\square$ Psychomotor performance unimpaired $^{4}$

$\checkmark$ Sedation similiar to placebo $^{5}$
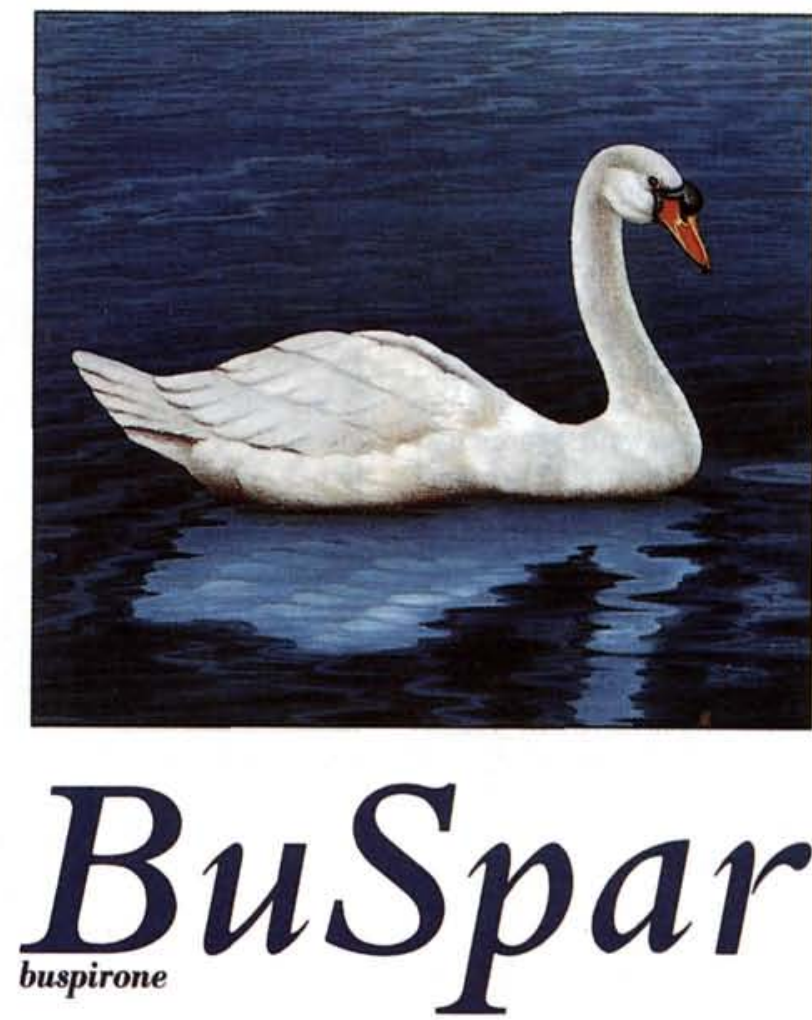

Anxiety therapy pure and simple 


\section{The Questions}

The research so far is more tantalising than illuminating. There are a number of questions which have not been adequately addressed, and a number of points of confusion.

\section{What is the difference between low cholesterol and lowered cholesterol?}

One important distinction concerns the difference between low cholesterol per se and lowered cholesterol. One reason why few prospective studies which examined the relationship between serum cholesterol and violent death have found an association may be that falling cholesterol, rather than low cholesterol, is the factor that results in lowered serotonergic turnover. If this is so, then a single measurement of cholesterol will not identify those at risk. In any event, the cholesterol levels being achieved in cholesterol-lowering trials, even those involving intensive pharmacotherapy, are still much higher than those studied by population studies of low cholesterol.

\section{Are there two different cause-effect relationships in the cholesterol-serotonin link?}

The next uncertainty arises from the possible interrelationships between cholesterol and serotonin. The first possible relationship is one in which lowered serotonin turnover lowers cholesterol by a mechanism as yet unknown. It could be, then, that the chronic low levels of cholesterol associated with suicide, aggression and impulsivity in psychiatric studies may be secondary to a neurotransmitter dysfunction. It is remotely unlikely that all individuals with low cholesterol have lower levels of impulse control, otherwise suicide would be more common in countries with low levels of cholesterol, such as the Mediterranean countries, and lowest in countries with high levels, such as Scandinavia.

The second possible relationship is where lowered cholesterol results in serotonergic dysfunction. Unlike the former case, it is unlikely that the serotonergic dysfunction would be permanent. It seems more plausible that other elements of serotonin metabolism could be up-regulated to compensate for the lowered receptor density and faster reuptake velocity. In this case, lowering cholesterol would open up a vulnerability 'window' in which the person's impulse control and mood would be adversely affected for an unknown period while serotonin activity was being rebalanced. In the World Health Organisation trial of clofibrate, although an increased mortality from non-cardiac causes was observed in the initial five years of the trial (24), this effect was not observed in the subsequent 8-year follow-up of the study group (25). We need further information on the long-term follow-up of patients on lipid-lowering drugs. It may well be that the effect on violent death is confined to the early period of treatment, though it is hard to imagine what mechanism may be responsible for a decline in risk over time.

It is noteworthy that the Family Health Study, in which dietary intervention was used over a 5 -year period to lower cholesterol, which used the Hopkins Symptom Checklist to assess depression and aggressive hostility, found that emotional state improved over the intervention period in the entire study group (26). Those who consumed a low-fat high complex-carbohydrate diet at the end of the study had significantly lower plasma cholesterols than those consuming a typical American diet, and they also showed a significantly greater improvement in both depression and hostility scores. Not alone does the study not show any evidence that dietary reduction of cholesterol is associated with negative emotional effects, if anything it has the opposite effect.

What are the confounding factors in the associations? The Whitehall study has shown that civil servants with low cholesterols were more likely to be in lower employment grades, to have had disease at time of cholesterol measurement, to have had a history of unexplained weight loss and to have been widowed (27). It may be that in prospective studies such confounding factors may drive apparent associations between low cholesterol and violent death.

It is unlikely, however, that the associations between cholesterol reduction and violent death are due to confounding variables, since they derive from studies in which subjects were randomised to receive either cholesterol-lowering interventions or placebo.

There has been criticism of studies linking psychiatric symptoms to cholesterol for their failure to allow for confounding variables. A recently-published study, however, which has shown such a link has also explored the possible confounding role of a wide variety of factors without managing to isolate any which removed the observed association (20).

\section{Case Proven?}

Assembling the necessary evidence to prove a cause-andeffect relationship in medicine is far from simple. Nevertheless, we should remember that it is not necessary to know the specific mechanism by which a risk factor works before establishing a causal relationship. The relationships between smoking and cancer or heart disease are taken as proven, despite the fact that we do not know exactly how the effect takes place. What is required first is a relaitonship of practical importance. This undoubtedly exists, as witnessed by the significant increases in violent death associated with both low and lowered cholesterol. To take a practical example, in the Lipid Research Clinics trial (28), there were 12 fewer deaths from coronary disease among those treated for elevated cholesterol (44 versus 32), but half of this benefit was lost to a higher rate of violent death - four in the control group, ten in the treated group. The second requirement is that of consistency. Here the picture is less clear. Results from drug trials of lipid lowering drugs have shown a more consistent effect on violent death than have dietary trials. Attempts to show links between low cholesterol and risk of violent death have been contradictory. Aside from the role of confounding factors, it may be that the risk of violent death is present only in a subgroup of those with low cholesterol. A third criterion is that the risk factor be present before the onset of the disease. Here we are faced with unanswered questions: do some psychiatric disorders cause low cholesterol and predispose to violent death? Certainly, though, the results of the cholesterol-lowering trials show that cholesterol change precedes increased risk of violent death in those treated with drugs.

A plausible mechanism (not necessarily proven) also 


\section{The one on the right is a modern Irish classic.}

Doctors take for granted the qualities that make

'Tagamet' a medicinal classic.

But many are unaware that $65 \%$ of the world's supply of the cimetidine for 'Tagamet' comes from Cork.*

That's what makes 'Tagamet' a modern Irish classic.

\section{TIMETIINE}

The only cimetidine ${ }^{\dagger}$ synthesised in Ireland.

Full prescribing information is available from Smith Kline \& French,

Corrig Avenue, Dun Laoghaire,

Co. Dublin.
Product authorisation holder:

Smith Kline \& French Laboratories Ltd Welwyn Garden City, Hertordshire,

England AL.7 1EY

- 1993 Smith Kline \& French Laboratories. Product authorisation number 60/6/1-6. Tagamet', Tiltab' and the appearance of the tablets are trade marks. tincludes other SB cimetidine-based products.

*The factory in Cork that synthesises cimetidine employs over 200 Irish people.

PRESCRIBING INFORMATION PRESENTATION Tagamet Tiltab' Tablets, PA 60/6/5, each containing $800 \mathrm{mg}$ cimetidine. 30 (rwo calendar strips of 15 tablets). 'Tagamer' Tablets, PA 60/6/4, each containing 400 mg cimetidine 60 (four calendar strips of 15 tablets). Tagamet Tiltab Tablets, PA 60/6/1, each containing $200 \mathrm{mg}$ cimetidine. 120 (four blister strips of 30 tabless). Tagamet' Syrup, PA $60 / 6 / 2$, containing $200 \mathrm{mg}$ cimetidine per $5 \mathrm{ml}$. $600 \mathrm{ml}$. Tagame, Injection, PA $60 / 6 / 3$, each ampoule containing $200 \mathrm{mg}$ cimetidine in $2 \mathrm{ml}$. 20 . Tagamer' infusion, PA $60 / 6 / 6$, containing $400 \mathrm{mg}$ cimetidine in $100 \mathrm{ml} 0.996 \mathrm{w} / \mathrm{vsodium}$ chloride. 20 . USES: Duodenal ulcer, benign gastric ukcer, recurren and stomal ulceration, reflux oesophagitis and other conditions where reduction of gastric acid is likely to be beneficial, Zollinger-Elison syndrome. DOSAGE. Usually oral, but parenteral or nasogastric route may be used. Usual

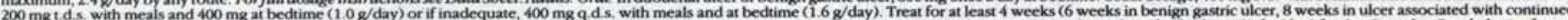
NSAIDs. To prevent relapse of peptic ulcer, usually $400 \mathrm{mg}$ at bedtime or else $400 \mathrm{mg}$ morning and at bedtime. Oesopbageal reflux disease: $400 \mathrm{mg} q$.ds. with meals and at bedtime (1.6 \&/day) for 4 to 8 weeks. Propbylaxis of stmess. induced gastrointestinal baemornbage, $200-400 \mathrm{mg}$ every $4-6$ hours, Propbylaxis of acid aspination symdrome, $400 \mathrm{mg} 90$-120 minutes before induction of general anaesthesia or at start of labour, up to $400 \mathrm{mg}$ repeated (parenterally

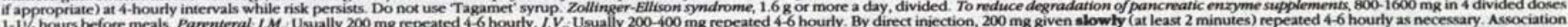
of cardiac arrhythmias with rapid injection. If there is cardiovascular impairment or if giving a larger dose, dilute and administer over at least 10 minutes. By intermittent infusion, the contents of one infusion bag infused over 30 minutes to 1 hour, repeated 4-6 hourly. By continuous infusion, usually $50-100 \mathrm{mg}$ hour over 24 hours. (See package inserts.) Elderly, As adult dosage, unless renal function markedly impaired. Colldren oover 2 years $25-30$. mp/kg/day, divided. CONTRA-INDICATION: Hypersensitivity to cimetidine. PRECAUTIONS: Impaired renal function: reduce dosage (see Data Sheet). Potentiation of oral anticoagulants, phenytoin and theophylline (see Data in patients with compromised bone marrow (see Data Sheet). Avoid during pregnancy and lactation. ADVERSE REACTIONS: Diarrihoea, dizziness, rash, tiredness. Gynaecomastia, occasional reversible liver damage, confusional states (usually in the elderly or very iil). Very rarely interstitial nephritis, acute pancreatitis, thrombocytopenia, agranulocytosis, headache, myalgia, arthralgia, sinus bradycardia, tachycardia, heart block. aplastic anaemia very rare reports of reversible impotence but no causal relationship established at usual therapeutic doses. OVERDOSAGE: No significant ill effects reported. Induction of vomiting or gastric lavage suggested with symptomatic and 
adds to the evidence for a causal effect. Serotonin seems the likeliest candidate at the moment, with the available evidence suggesting connexions between cholesterol concentration and serotonergic function which would result in lowered impulse control and depressive symptoms. So far there is no data to suggest a graded association between cholesterol and violent death, and the reversal of risk with the removal of the risk factor, that other component of a causal relationship, cannot be investigated without considerably more preliminary evidence than we have to hand.

Finally, the quality of the research to date is variable; it has largely involved data accumulated for other purposes, and some of the measure used are, at best, proxy measures. This applies not alone to extrapolation of platelet serotonin behaviour to CNS activity, but also applies to the diverse methods used to measure personality and psychopathological variables. What we urgently need is well-designed prospective research.

\section{The Practical Implications}

Although drug treatment of high cholesterol increases risk of violent death by about $75 \%$, the absolute risk of violent death is lower than the risk of cardiovascular death. It is likely that, especially with the latest generation of cholesterol-lowering drugs, the reduction in cardiovascular mortality will lead to a reduction in overall mortality, even with a slight increase in violent death and in deaths due to some cancers among those treated. It is worth bearing in mind, however, that in the most recent meta-analyses of prevention trials, dietary intervention to reduce cholesterol was associated with a reduction in cardiovascular disease that was similar to that seen in drug trials, but was not associated with increased risk of violent death. Dietary reduction of cholesterol seems to offer the benefits of drug treatment without the drawback of an increased rate of violent death $(29,30)$. In contrast to the reports of depression associated with drug treatment of cholesterol is the recent report from the Family Heart Study referred to above, which showed positive mood changes associated with dietary reduction of cholesterol. (26)

Why should the use of drugs to lower cholesterol result in negative emotional symptoms and an increased risk of violent death, while the use of diet appears to result in neither? The range of drugs used to lower cholesterol argues against a simple drug side-effect. The speed at which drugs lower cholesterol could be a factor, but the duration of the effect (at least five years) suggests that there is some common feature to cholesterol-reducing drugs involved in the effect.

On a practical level, the increased reporting of depressive symptoms in patients receiving cholesterollowering therapy should alert medical personnel to the need to monitor mood in such patients and to consider cholesterol-lowering drugs as at least a contributory factor in cases of depression. Theoretically, at least, patients on drug treatment for cholesterol may also display lower levels of impulse control. We urgently need prospectively-gathered information on the effect of cholesterol therapy on mood and function. It may be necessary to monitor these in patients to detect adverse reactions.

A central lession in all of this, however, is one which is hard to learn, no matter how many good examples we encounter: it is that all medical treatments have a potential to affect the psychological function of the patient. By confining our attention to the treatment or prevention of specific diseases, we risk ignoring the impact of our efforts on the patient's life, both qualitatively and quantitatively.

\section{References}

1. Muldoon M, Manchuch S, Mathews K. Lowering cholesterol concentrations and mortality: a quantitative review of primary prevention trials. BMJ 1990; 301: 309-14.

2. Lipid Clinics Research Program. The lipid research clinics coronary prevention trial results. JAMA 1984; 251: 351-74.

3. Virkkunen $M$. The lipid research clinics coronary prevention trial results [letter]. JAMA 1985; 253: 635-6.

4. Pekkanen J, Nissinen A, Punsar S, Karvonen M. Serum cholesterol and risk of accidental or violent death in a 25-year follow-up. Arch Intern Med 1989; 149: 1589-91.

5. Asberg M, Traskman L, Thoren P. 5-HIAA in cerebrospinal fluid: a biochemical suicide predictor. Arch Gen Psychiatry 1976; 33: 93-7.

6. Anon. Serotonin, suicidal behaviour and impulsivity. Lancet 1987; 2: 949-50.

7. Lindberg G, Rästam L, Gullberg B, Eklund G. Low serum cholesterol concentration and short term mortality from injuries in men and women. BMJ 1992; 305: 277-9.

8. Kromhout D, Katan M, Menotti A, Keyes A, Blomberg B. Serum cholesterol and long-term death rates from suicide, accidents or violence [letter]. Lancet 1992; 340: 317.

9. Jacobs D, Blackburn H, Higgins $M$, et al. Report of the conference on low blood cholesterol: mortality associations. Circulation 1992; 86: 1046-60.

10. Schuit A, Dekker J, Schouten E, Kok F. Low serum cholesterol and death due to accidents, violence, or suicide. Lancet 1993; $341: 827$.

11. Marmot M, Davey Smith G, Stansfeld S, et al. Health inequalities among British civil servants: the Whitehall II study. Lancet 1991; 337; 1387-93.

12. Lewis B, Katan M, Merkx I, et al. Towards an improved lipid-lowering diet: aditive effects of changes in nutrient intake. Lancet. 1981; ii: 1310-3.

13. Conroy R. Serum triglycerides and aggression in the general population. [letter]. Lancet 1993; 341: 176.

14. Strandbert T, Valvanne J, Tilvis R. Low serum lipids and depression. Lancet $1993 ; 341: 433-4$

15. Preston Mason $R$, Herbette L, Silverman D. Can altering serum cholesterol affect neurologic function? J Mol Cell Cardiol 1991; 23: $1339-42$.

16. Engelberg H. Low serum cholesterol and suicide. Lancet 1992; 339: 727-9.

17. Guicheney P, Devynck MA, Cloix JF, Pernollet MG, Grichois ML, Meyer P. Platelet 5-HT content and uptake in essential hypertension: rôle of endogenous dititalis-like factors and plasma cholesterol. J Hypertens 1988; 6: 115-26.

18. Spoont M. Modulatory rôle of serotonin in neural informaito processing: implications for human psychopathology. Psych Bull 1992; 112: 330-50.

19. Morgan R, Palinkas L, Barrett-Connor E, Wingard D. Plasma cholesterol and depressive symptoms in older men. Lancet 1993; 341: 75-9.

20. Palinkas L, Barrett-Connor E, Morgan R, Wingard D. Serum lipids and depression. Lancet 1993; 341: 563 .

21. Davey Smith G, Shipley M. Serum lipids and depression Lancet 1993; 341: 434.

22. DeAlberto $M$, Duicimitière $P$, Mailard $P$, Alperovitch $A$. Serum lipids and depression Lancet 1993; $341: 435$.

23. Duits N, Bos FM. Depressive symptoms and cholesterol-lowering drugs [letter]. Lancet 1993; 341: 114.

24. Report from the Committee of Principal Investigators. A cooperative trial in the primary prevention of ischaemic heart disease using clofibrate. Br Heart J 1978; 40: 1069-118.

25. Report from the Committee of Principal Investigators. WHP cooperative trial on primary prevention of ischaemic heart diesease with clofibrate to lower serum cholesterol: final mortality follow-up. Lancet 1984; ii: 600-04.

26. Weidner $\mathrm{G}$, Connor $\mathbf{S}$, Hollis J, Connor W. Improvements in hostility and depression in relation to dietary change and cholesterol lowering. Ann Intern Med 1992; 117: 820-3.

27. Davey Smith G, Shipley MJ, Marmot MG, Rose G. Plasma cholesterol concentration and mortality. JAMA 1992; 267: 70-6.

28. Lipid Clinics Research Program. The Lipid Clinics Primary Prevention Trial results: I Reduction in incidence of coronary heart disease. JAMA 1984; 251: 351-74.

29. Holme I. Relation of coronary heart disease incidence and total mortality to plasma cholesterol reduction in randomised trials: use of meta-analysis. Br Heart J 1993; 69: S42-S47.

30. Davey Smith G, Song F, Sheldon T. Cholesterol lowering and mortality: the importance of considering initial level of risk. BMJ 1993; 306: 1357-73. 


\section{Sleep tight。}

\section{.Wake bright}
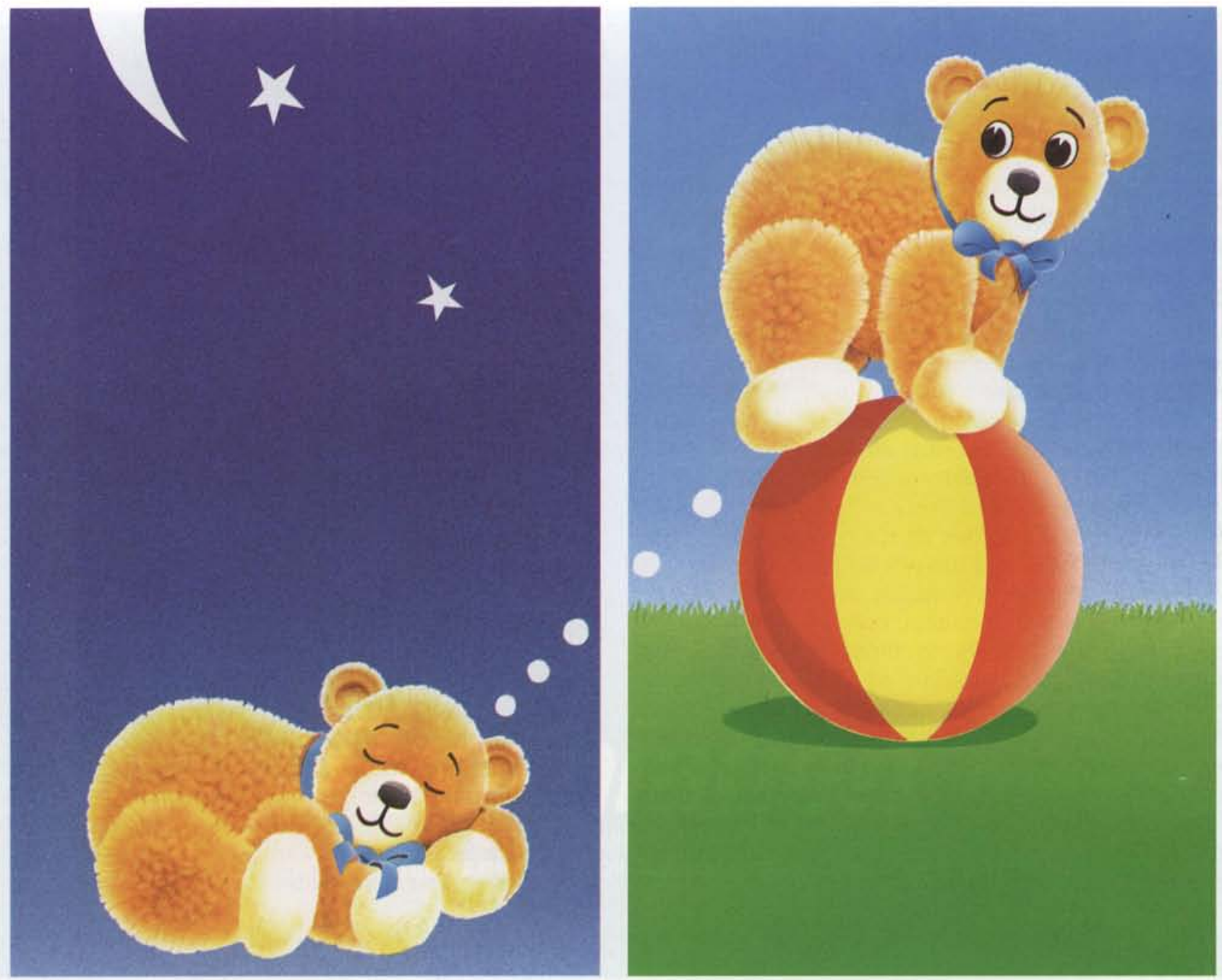

\section{Zimovane \\ zopiclone}

\section{When patients need to be on the ball}

For prescribing and further information please contact Rhône-Poulenc Rorer Ireland Ltd., 14 Deansgrange Industrial Estate, Blackrock, Co. Dublin. PA: 40/78/1

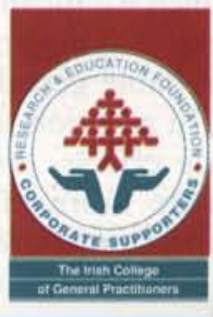

